\title{
Guest Editorial: Selected Papers from European Symposium on Algorithms
}

\author{
Dan Halperin · Kurt Mehlhorn
}

Published online: 30 March 2010

(C) Springer Science+Business Media, LLC 2010

Issue is dedicated to a selection of papers from the 16th Annual European Symposium on Algorithms (ESA) held in Karlsruhe, Germany, September 15-17, 2008. Five papers were invited from the "Design and Analysis Track" and three from the "Engineering and Applications Track"; seven papers were submitted and went through the standard refereeing process of Algorithmica.

The paper "Better and Simpler Approximation Algorithms for the Stable Marriage Problem" by Zoltan Kiraly received the best paper award. It introduces a new approximation algorithm for the stable marriage problem. The algorithm improves on its predecessors on all accounts: simplicity, quality of approximation, and efficiency.

The contribution "Deterministic Sampling Algorithms for Network Design" by Anke van Zuylen received the best student paper award in the design and analysis track. For several NP-hard network design problems, the best known approximation algorithms follow a simple randomized strategy, called sample-and-augment. A random sample from the input is drawn, a certain problem is solved, and the solution is extended to a full solution. Anke shows how to derandomize these algorithms by exhibiting a specific sample that works well.

Hervé Fournier and Antoine Vigneron address a basic approximation problem: "Fitting a Step Function to a Point Set". Given an integer $k$ and a set $P$ of points in the plane, the goal is to find the step function that minimizes the maximal vertical distance between the point set and the step function. The authors present an algorithm that runs in time $O(n)$ for a point set sorted by $x$-coordinate.

D. Halperin

Department of Computer Science, Tel Aviv University, Tel Aviv, Israel

K. Mehlhorn ( $₫)$

Max-Planck-Institut für Informatik, Saarbrücken, Germany

e-mail: mehlhorn@mpi-inf.mpg.de 
In "An Almost Space-Optimal Streaming Algorithm for Coresets in Fixed Dimensions", Hamid Zarrabi-Zadeh presents a new streaming algorithm for maintaining the $\epsilon$-kernel of a point set. The space requirement of the algorithm is optimal up to a small logarithmic factor. The algorithm implies improved algorithms for a number of geometric problems in fixed dimensions, e.g., width of a point set, minimum enclosing cylinder, and minimum enclosing annulus.

The next contribution, Time-Dependent SHARC-Routing by Daniel Delling, is the recipient of the best student paper award for the "Engineering and Applications Track." The author presents practical algorithms for computing time-dependent shortest paths on road and rail networks. The techniques used by the author generalize elements from previous papers, which, due to the time dependence become more much complicated, and a good deal of engineering is required to obtain reasonable running times. The author succeeds in doing so, with impressive results.

The paper "Coupled Path Planning, Region Optimization, and Applications in Intensity-modulated Radiation Therapy" by Danny Z. Chen, Shuang Luan, and Chao Wang, addresses an optimization problem that arises, as the title suggests, in radiation therapy. The solutions proposed in the paper combine new ideas using the geometry and graph structure of the problem at hand, and substantially improve over solutions based on standard techniques. The end result is a control sequence for a specific radiation-therapy machine, which is essential to optimally deliver a precisely prescribed radiation dose, while minimizing the side-effects on the surrounding healthy tissue.

This issue concludes with the contribution "An Integer Programming Algorithm for Routing Optimization in IP Networks by Andreas Bley." The author presents an integer-programming algorithm to optimize the routing in communication networks based on shortest path routing protocols, which are widely used in the Internet. After analyzing the problem from a theoretical point of view, the author describes the new approach, discusses major implementation issues and reports computational results for various benchmark problems, which demonstrate the efficiency of the approach.

We would like to thank the authors for contributing to this special issue and the referees for their attentive and helpful work in the review process.

\section{Guest Editors}

